\title{
Abstract: Automatic Dementia Screening and Scoring by Applying Deep Learning on Clock-drawing Tests
}

\author{
Shuqing Chen ${ }^{1}$, Daniel Stromer ${ }^{1}$, Harb Alnasser Alabdalrahim ${ }^{1}$, \\ Stefan Schwab ${ }^{2}$, Markus Weih ${ }^{2}$, Andreas Maier ${ }^{3}$ \\ ${ }^{1}$ Pattern Recognition Lab, Computer Science, FAU Erlangen-Nürnberg \\ ${ }^{2}$ Department of Neurology, FAU Erlangen-Nürnberg \\ ${ }^{3}$ Pattern Recognition Lab, FAU Erlangen-Nürnberg \\ shuqing. chen@fau.de
}

\begin{abstract}
Dementia is one of the most common neurological syndromes in the world. Usually, diagnoses are made based on paper-and-pencil tests and scored by personal judgments of experts. This technique can introduce errors and has high inter-rater variability. In this study, the an automatic assessment of clockdrawing test (CDT) images was developed [1]. The CDTs were classified by the Shulmann system with scores varying from 1 to 6 , increasing accordingly to the severity of dementia. To automatically classify the CDTs, our work is based on a comparison of three deep learning architectures including transfer learning of three pre-trained nets: VGG16, ResNet-152, and DenseNet-121. The dataset for our experiments consisted of 1315 individuals. To avoid the bias caused by the random data selection for the training with the limited amount of data, which also included several dementia types, we selected the training data and the validation data based on manifold learning methods. For training the network, we used optimization strategies such as adaptive moment estimation, stochastic gradient descent, root mean squared probability and a learning-rate scheduler. We discovered that using a varying learning rate yields in better results compared to a fixed learning rate during training. The outcome of our work is a standardized and digital estimation of the dementia screening result and severity level for an individual. We achieved off-diagonal accuracies of $96.65 \%$ for screening and up to $98.54 \%$ for scoring. Our proposed neural network achieved very high AUC and outperformed reported clinical screening results by up to $27 \%$, other machine-learning screening techniques by up to $24 \%$, and machinelearning scoring approaches up to $27 \%$. The algorithm can be easily integrated into hospitals' environments and care facilities to help monitoring the state of patients with dementia.
\end{abstract}

\section{References}

1. Chen S, Stromer D, Alabdalrahim HA, et al. Automatic dementia screening and scoring by applying deep learning on clock-drawing tests. Sci Rep. 2020;10(20854):111. 